\title{
More Monotonicity Theorems for Partitions
}

\author{
Jane Friedman, James T. Joichi and Dennis Stanton
}

\section{CONTENTS}

1. Introduction

2. The Conjecture

3. Monotone Subsets

4. Injections

5. Related Questions
Consider the collection of all integer partitions whose part sizes lie in a given set. Such a set is called monotone if the generating function has weakly increasing coefficients. The monotone subsets are classified, assuming an open conjecture.

\section{INTRODUCTION}

Suppose $P$ is a set of positive integers, and let $a_{n}$ be the number of integer partitions of $n$ whose part sizes lie in $P$. It is well known [Andrews 1976] that the generating function for the sequence $a_{n}$ is

$$
F_{P}(q)=\sum_{n=0}^{\infty} a_{n} q^{n}=\prod_{p \in P} \frac{1}{1-q^{p}} .
$$

Bateman and Erdös [1956] found necessary and sufficient conditions on $P$ so that the $k$-th difference of the sequence $a_{n}$ is asymptotically positive. In this paper we consider $k=1$. We seek a stronger conclusion, namely that $a_{n+1} \geq a_{n}$ for all $n>1$, or, equivalently, that all of the coefficients in $(1-q) F_{P}(q)$ past the linear term are nonnegative. A set $P$ of positive integers satisfying this condition is called monotone.

Let $n$ be the smallest element of $P$. Clearly any $P$ containing 1 is monotone, so we can assume $n>1$. If $P$ is monotone, the coefficient of $q^{n+1}$ in $(1-q) F_{P}(q)$ is nonnegative, and we must have $n+1 \in P$. In fact, it is easy to see in this way that $\{n, \ldots, 2 n-1\} \subset P$. In Theorems 3.5 and 3.6 we classify all monotone $P$ with $n \geq 6$, assuming Conjecture 2.2 below.

A set $P$ is called asymptotically alternating if there exists a large enough $k$ so that the $k$-th differences of $a_{n}$ alternate in sign. We classify the asymptotically alternating sets $P$ in Theorem 5.2. 
We shall use $\mathrm{NN}$ to denote nonnegative terms in a power series $F(q)$, and $\operatorname{SPP}\left(q^{a}\right)$ to denote strictly positive terms past $q^{a}$. For example, we have $-q+$ $1 /\left(1-q^{2}\right)=1-q+\mathrm{NN}$ and $q^{3} /(1-q)=\operatorname{SPP}\left(q^{2}\right)$.

\section{THE CONJECTURE}

In this section we concentrate on properties of the function

$$
f_{n, m}(q)=\frac{1-q}{\prod_{i=n}^{m}\left(1-q^{i}\right)} .
$$

We formulate a conjecture on $f_{n, m}(q)$ below, and use it to classify monotone sets $P$ in Section 3 . We are particularly interested in the values of $m$ for which $f_{n, m}(q)=1-q+\mathrm{NN}$, because $P=$ $\{n, \ldots, m\}$ is monotone for such $m$.

Proposition 2.1. We have

$f_{n, \infty}(q)=1-q+\mathrm{NN}=1-q+\mathrm{NN}+\operatorname{SPP}\left(q^{3 n+1}\right)$.

Proof. Applying the $q$-binomial theorem [Andrews 1976], we obtain

$$
\begin{aligned}
f_{n, \infty}(q) & =(1-q) \sum_{k=0}^{\infty} \frac{q^{n k}}{\prod_{i=1}^{k}\left(1-q^{i}\right)} \\
& =1-q+q^{n}+\frac{q^{2 n}}{1-q^{2}}+\sum_{k=3}^{\infty} \frac{q^{n k}}{\prod_{i=2}^{k}\left(1-q^{i}\right)} \\
& =1-q+\mathrm{NN}+\operatorname{SPP}\left(q^{3 n+1}\right) .
\end{aligned}
$$

Conjecture 2.2. If $n>1$ is an odd integer, we have $f_{n, 2 n-1}(q)=1-q+\mathrm{NN}$. If, in addition, $n \geq 7$, then

$$
f_{n, 2 n-1}(q)=1-q+\mathrm{NN}+\operatorname{SPP}\left(q^{3 n+4}\right) .
$$

If $n>1$ is even, then

$$
f_{n, 2 n+1}(q)=1-q+\mathrm{NN}+\operatorname{SPP}\left(q^{3 n+7}\right) .
$$

It is easy to see that the even part of this conjecture follows from the odd part. If $h_{n, m}(q)=f_{n, m}(q)-$ $1+q=\mathrm{NN}$, then

$$
h_{n, 2 n+1}(q)=\frac{1}{1-q^{n}}\left(h_{n+1,2 n+1}(q)+q^{n}-q^{n+1}\right) .
$$

If $n$ is even, $h_{n+1,2 n+1}(q)$ contains $q^{n+1}$. This shows that $h_{n, 2 n+1}(q)=\mathrm{NN}$, and that $h_{n, 2 n+1}(q)=\mathrm{NN}+$
$\operatorname{SPP}\left(q^{3 n+7}\right)$ for $n \geq 6$. One can prove the cases $n=2$ and $n=4$ separately.

A natural way to prove Conjecture 2.2 for a given $n$ is to use the asymptotics to verify the large coefficients, and check the small coefficients separately. For this one needs an effective bound for the positivity of the large coefficients. In turns out that a recurrence relation can find this effective bound empirically, using Mathematica or a traditional programming language.

Proposition 2.3. Conjecture 2.2 holds for $n \leq 37$.

Proof. We verify the case of $n$ odd. Let $a_{k}(n, n+i)$ denote the number of partitions of $k$ into parts of size $n, \ldots, 2 n-1$ whose largest part is $n+i$, for $0 \leq i \leq n-1$. We must show that

$$
\delta(k)=\sum_{i=0}^{n-1}\left(a_{k}(n, n+i)-a_{k-1}(n, n+i)\right) \geq 0
$$

for $k \geq 2$. By removing this largest part, we have

$$
a_{k}(n, n+i)=\sum_{j=0}^{i} a_{k-n-i}(n, n+j) .
$$

Suppose that, by applying $(2.1)$ recursively to $\delta(k)$, we can express $\delta(k)$ as the sum of $\delta(k-t)$ with a nonnegative linear combination of $a_{j}(n, n+i)$ 's. If we verify that $\delta(2), \ldots, \delta(t+1) \geq 0$, then Conjecture 2.2 holds.

For example, if $n=3$, we have

$$
\delta(k)=\delta(k-20)+a_{k-21}(3,3),
$$

and we check that $\delta(2), \ldots, \delta(21) \geq 0$. This is feasible as long as $t=t(n)$ does not grow too rapidly with $n$. Empirically, we find $t(n)=(2 n-2)(2 n-1)$. If the smallest part is used to generate a recurrence analogous to (2.1), the empirical result is $t(n)=n(n+1)$ if 3 does not divide $n$, otherwise $t(n)=4 n(n+1)$. In this way Conjecture 2.2 was verified for $n \leq 37$.

We shall need the lemma below in the next section.

Lemma 2.4. If $n \geq 7$ is an odd integer, the coefficient of $q^{6 n+1}$ in $f_{n, 2 n-1}(q)$ is at least 2 . 
Proof. The $q$-binomial theorem implies that

$$
f_{n, 2 n-1}(q)=1-q+\sum_{k=1}^{\infty}\left[\begin{array}{c}
n+k-1 \\
k
\end{array}\right]_{q}(1-q) q^{n k} .
$$

The only terms in this expression that contribute to $q^{6 n+1}$ are $k=4$ and $k=5$. It is easy to see that the desired coefficient is equal to the coefficient of $q^{2 n+2}$ in

$$
\begin{aligned}
\frac{q}{\left(1-q^{2}\right)\left(1-q^{3}\right)\left(1-q^{4}\right)}-\frac{1}{\left(1-q^{2}\right)\left(1-q^{4}\right)\left(1-q^{6}\right)} \\
+\frac{1}{\left(1-q^{4}\right)\left(1-q^{6}\right)\left(1-q^{8}\right)\left(1-q^{10}\right)}
\end{aligned}
$$

An elementary injection shows that this coefficient is at least 2 , for $n \geq 7$ odd. We do not give the details.

\section{MONOTONE SUBSETS}

In this section we use Conjecture 2.2 to classify the monotone subsets $P$, in Theorems 3.5 and 3.6. For most of this section we shall assume that $n$ is odd. Basically we need a method to change the set $P$ from an interval $\{n, \ldots, 2 n-1\}$ to a larger class of sets. The lemma below accomplishes this.

Lemma 3.1. Suppose that

$$
H(q)=1-q+\mathrm{NN}+\operatorname{SPP}\left(q^{a}\right),
$$

and

$$
H(S, q)=\frac{H(q)}{\prod_{s \in S}\left(1-q^{s}\right)},
$$

where $S$ is any set of positive integers. If $s \geq a$ for all $s \in S$, then $H(S, q)=1-q+\mathrm{NN}$.

Proof. We must show that $g(S, q)=H(S, q)-1+$ $q=$ NN. An easily verified recurrence for $b \notin S$ is

$$
g(S \cup\{b\}, q)=\frac{1}{1-q^{b}}\left(g(S, q)+q^{b}-q^{b+1}\right) .
$$

For $S$ the empty set, $g(\varnothing, q)$ is positive past $q^{a}$, thus positive at $q^{b+1}$. So (3.1) implies $g(\{b\}, q)=$ $\mathrm{NN}+\operatorname{SPP}\left(q^{b+1}\right)$, and the argument follows for finite $S$ by induction on $|S|$. If $S$ is infinite, to check that the coefficient of $q^{j}$ is nonnegative, we apply the finite part this lemma for the finite set $S \cap\{x: x \leq j\}$.

We next find monotone sets $P$ from Lemma 3.1 and Conjecture 2.2.

Proposition 3.2. If Conjecture 2.2 is true, then $P=$ $\{n, \ldots, 2 n-1\} \cup Q$ is monotone, where $n \geq 7$ is odd and $Q$ is any subset of $\{3 n+4,3 n+5, \ldots\}$.

Suppose that $P$ is monotone and satisfies $\{n, \ldots$, $2 n-1\} \subset P \subset\{n, \ldots, 3 n-2\}$. It is easy to see that if an even number $e \geq 2 n$ is in $P$, then so is $e+1$. The next proposition shows that this condition characterizes such monotone sets $P$.

Proposition 3.3. If Conjecture 2.2 is true, then $P=$ $\{n, \ldots, 2 n-1\} \cup E \cup O$ is monotone, where $n \geq 7$ is odd, $E$ and $O$ are subsets of $\{2 n, \ldots, 3 n-2\}$ containing even and odd elements, respectively, and the translate $E+1$ is contained in $O$.

Proof. Suppose $n \geq 7$ is odd, and put, for any subset $S \subset\{2 n, 2 n+1, \ldots\}$,

$$
g(n, S)=\frac{f_{n, 2 n-1}(q)}{\prod_{s \in S}\left(1-q^{s}\right)}-1+q .
$$

The coefficients of $f_{n, 2 n-1}(q)$ up to $q^{3 n+4}$ can be explicitly found, so that Conjecture 2.2 implies that

$$
g(n, \varnothing)=q^{n}+\sum_{i=0}^{(n-3) / 2} q^{2 n+2+2 i}+q^{3 n+3}+\operatorname{SPP}\left(q^{3 n+4}\right) .
$$

To go from $g(n, \varnothing)=\mathrm{NN}$ to $g(n, E \cup O)=\mathrm{NN}$ we add either a single odd $a$ or consecutive entries $a$ and $a+1$, one being even and the other odd. In the first case we have

$$
g(n, S \cup\{a\})=\frac{q^{a}-q^{a+1}+g(n, S)}{1-q^{a}},
$$

for $a \notin S$, while in the second we have

$$
g(n, S \cup\{a, a+1\})=\frac{q^{a}}{1-q^{a}}+\frac{q^{2 a+2}-q^{a+2}+g(n, S)}{\left(1-q^{a}\right)\left(1-q^{a+1}\right)}
$$

for $a, a+1 \notin S$. We see from (3.2) that $g(n, \varnothing)$ contains all of the even powers of $q$ from $2 n+2$ to 
$3 n-1$. If $a$ is odd, (3.3) implies that $g(n,\{a\})$ is nonnegative and contains all of the even powers of $q$ from $a+2$ to $3 n-1$. If the smallest element $a$ of $E \cup O$ is even, (3.4) implies that $g(n,\{a, a+1\})$ is nonnegative and contains all of the even powers of $q$ from $a+3$ to $3 n-1$. We continue by induction, noting that the single negative term in (3.3) and (3.4) is an even power past the new term which is added, and thus is always cancelled. All of the other coefficients must increase. We obtain

$$
g(n, E \cup O)=\mathrm{NN}+\operatorname{SPP}\left(q^{3 n+4}\right)
$$

for $n \geq 7$ odd.

Proposition 3.4. If Conjecture 2.2 is true and $n \geq 7$ is odd, then $P=\{n, \ldots, 2 n-1\} \cup E \cup O \cup Q$ is monotone, where $E, O$, and $Q$ are in Propositions 3.2 and 3.3 .

Proof. This follows from (3.5) and Lemma 3.1.

Suppose we generalize Proposition 3.4 to the case where $n \geq 7$ is odd, $P=\{n, \ldots, 2 n-1\} \cup E \cup O \cup A$, and $A \subset\{3 n-1,3 n, 3 n+1,3 n+2,3 n+3,3 n+4\}$. From (3.5) we know that

$$
g(n, E \cup O)=\mathrm{NN}+\operatorname{SPP}\left(q^{3 n+4}\right) .
$$

We will use (3.3), (3.4), and analogous versions for three and four in a row (see (3.6) and (3.7) below) to conclude that

$$
g(n, E \cup O \cup A)=\mathrm{NN}+\operatorname{SPP}\left(q^{3 n+4}\right),
$$

for the appropriate sets $A$.

From (3.2) there is exactly one term in $g(n, \varnothing)$ from $q^{3 n}$ to $q^{3 n+4}$, namely $+q^{3 n+3}$. For $g(n, E \cup$ $O \cup A$ ), the possible new partitions in this range whose differences we must take are:

- $\{3 n-1\}$,

- $\{3 n\},\{n, 2 n\}$,

- $\{3 n+1\},\{n, 2 n+1\},\{n+1,2 n\}$,

- $\{3 n+2\},\{n, 2 n+2\},\{n+1,2 n+1\},\{n+2$, $2 n\}$,

- $\{3 n+3\},\{n, 2 n+3\},\{n+1,2 n+2\},\{n+2$, $2 n+1\},\{n+3,2 n\}$,
- $\{3 n+4\},\{n, 2 n+4\},\{n+1,2 n+3\},\{n+2$, $2 n+2\},\{n+3,2 n+1\},\{n+4,2 n\}$.

If $3 n-1 \in A$, nonnegativity implies either $2 n \in E$ or $3 n \in A$. If $2 n \in E$ we use (3.3) with $a=$ $3 n-1$ to get strict positivity past $3 n+4$. The case where $3 n-1 \notin A$ and $3 n \in A$ is done by the same argument. If $3 n-1$ and $3 n \in A$ we use (3.4), and must check the coefficient of $q^{3 n+1}$ in $g(n, E \cup O \cup\{3 n-1,3 n\})$. Again we must have either $3 n+1 \in A$ or $2 n+1 \in O$. If $2 n+1 \in O$, then (3.4) gives strict positivity past $3 n+4$. Otherwise $3 n-1,3 n, 3 n+1 \in A$, and we use $g(n, E \cup O \cup\{a, a+1, a+2\})$

$$
\begin{aligned}
= & \frac{q^{a}}{\left(1-q^{a}\right)\left(1-q^{a+2}\right)}+\frac{q^{2 a+4}}{\left(1-q^{a+1}\right)\left(1-q^{a+2}\right)} \\
& +\frac{q^{3 a+3}-q^{a+3}+g(n, E \cup O)}{\left(1-q^{a}\right)\left(1-q^{a+1}\right)\left(1-q^{a+2}\right)}
\end{aligned}
$$

for $a=3 n-1$. If $2 n+2 \in E$, the term $q^{3 n+2}$ appears in $g(n, E \cup O)$, and (3.6) implies that

$g(n, E \cup O \cup\{3 n-1,3 n, 3 n+1\})=\mathrm{NN}+\operatorname{SPP}\left(q^{3 n+4}\right)$.

If $2 n+2 \notin E$, clearly we must have $3 n-1,3 n$, $3 n+1,3 n+2 \in A$. This time we use

$$
\begin{aligned}
g(n, E & \cup O \cup\{a, a+1, a+2, a+3\}) \\
= & \frac{q^{a}}{\left(1-q^{a}\right)\left(1-q^{a+2}\right)\left(1-q^{a+3}\right)} \\
& +\frac{q^{2 a+4}+q^{3 a+3}}{\left(1-q^{a}\right)\left(1-q^{a+1}\right)\left(1-q^{a+2}\right)} \\
& +\frac{q^{2 a+6}+q^{4 a+7}-q^{a+4}-q^{2 a+3}+g(n, E \cup O)}{\left(1-q^{a}\right)\left(1-q^{a+1}\right)\left(1-q^{a+2}\right)\left(1-q^{a+3}\right)}
\end{aligned}
$$

for $a=3 n-1$. Since $g(n, \varnothing)$ contains $q^{3 n+3}$, the $q^{a+4}$ term is cancelled in (3.7). From Lemma 2.4, $g(n, \varnothing)$ contains at least $+2 q^{6 n+1}$; thus $g(n, E \cup O)$ does also, and (3.7) implies that

$$
\begin{aligned}
g(n, E \cup O \cup\{3 n-1,3 n, 3 n & +1,3 n+2\}) \\
& =\mathrm{NN}+\operatorname{SPP}\left(q^{3 n+4}\right) .
\end{aligned}
$$

Finally, if $3 n+3 \in A$, we must have either $2 n+4 \in$ $E$ or $3 n+4 \in A$, and we retain strict positivity past $q^{3 n+4}$. 
Applying Lemma 3.1, we have proved the main theorem.

Theorem 3.5. If Conjecture 2.2 is true, the monotone subsets $P$ whose minimum value $n$ is odd, $n \geq 7$, are $P=\{n, \ldots, 2 n-1\} \cup E \cup O \cup A \cup Q$, where

- $E$ is a set of even integers from $\{2 n, \ldots, 3 n-2\}$,

- $O$ is a set of odd integers from $\{2 n, \ldots, 3 n-2\}$ such that $E+1 \subset O$,

- $A$ is a subset of $\{3 n-1, \ldots, 3 n+3\}$ such that if $3 n+i \in A$ for $i \neq 2$, then either $3 n+i+1 \in P$ or $2 n+i+1 \in P$, and

- $Q$ is a subset of $\{3 n+4,3 n+5, \ldots\}$.

For even values of $n$, the coefficient of $q^{3 n+1}$ easily implies $2 n+1 \in P$. The analog of (3.2) for $n \geq 6$ that follows from Conjecture 2.2 is

$$
\begin{aligned}
\frac{f_{n, 2 n-1}(q)}{1-q^{2 n+1}}=1- & q+q^{n}+q^{2 n+1}+\sum_{i=0}^{n / 2} q^{2 n+4+2 i} \\
& +2 q^{3 n+6}+\operatorname{SPP}\left(q^{3 n+7}\right)
\end{aligned}
$$

A complicated injection proves that (3.8) also holds for $n=4$. Completely analogous arguments based upon (3.8) yield the next theorem. We do not need an even version of Lemma 2.4, because the largest gap in (3.8) from $3 n+2$ to $3 n+6$ has width 2 , not width 4 , as in (3.2).

Theorem 3.6. If Conjecture 2.2 is true, the monotone subsets $P$ whose minimum value $n$ is even, $n \geq 4$, are $P=\{n, \ldots, 2 n-1,2 n+1\} \cup E \cup O \cup$ $A \cup Q$, where

- $E$ is a set of even integers from $\{2 n+2, \ldots$, $3 n+1\}$,

- $O$ is a set of odd integers from $\{2 n+2, \ldots$, $3 n+1\}$ such that $E+1 \subset O$,

- $A$ is a subset of $\{3 n+2, \ldots, 3 n+6\}$ such that if $3 n+2 i \in A$ then either $3 n+2 i+1 \in P$ or $2 n+2 i+1 \in P$, and

- $Q$ is a subset of $\{2 n, 3 n+7,3 n+8, \ldots\}$.

Propositions 2.1, 3.2 and 3.3 imply that $f_{n, m}(q)=$ $1-q+\mathrm{NN}$ if
- $m \in\{2 n-1,2 n+1,2 n+3, \ldots, 3 n-2,3 n-1$, $3 n, \ldots, \infty\}$ for $n$ odd, or

- $m \in\{2 n+1,2 n+3,2 n+5, \ldots, 3 n+1,3 n+2$, $3 n+3, \ldots, \infty\}$ for $n$ even.

We cannot prove a weaker version, namely that for any fixed $n$, there is some finite $m$ for which $f_{n, m}(q)=1-q+\mathrm{NN}$. Nonetheless, we do have the following result:

Proposition 3.7. Suppose $n$ is odd. If $f_{n, m}(q)=1-$ $q+\mathrm{NN}$ for some $m=m_{0} \geq 3 n-2$, then $f_{n, m}(q)=$ $1-q+\mathrm{NN}$ for all $m>m_{0}$.

Proof. Upon adding $m_{0}+1$, from (3.1), we need only show that the coefficient of $q^{m_{0}+2}$ in $f_{n, m_{0}}(q)-1+q$ is $\geq 1$. But this coefficient equals the coefficient of $q^{m_{0}+2}$ in $f_{n, \infty}(q)$. From the proof of Proposition 2.1 we have

$f_{n, \infty}(q)=1-q+q^{n}+\frac{q^{2 n}}{1-q^{2}}+\frac{q^{3 n}}{\left(1-q^{2}\right)\left(1-q^{3}\right)}+\mathrm{NN}$, and any term past $q^{3 n+1}$ appears with coefficient $\geq$ 1 in the fourth summand. Since $n$ is odd, $q^{3 n+1}$ and $q^{3 n-1}$ appear in the third summand. Clearly $q^{3 n}$ appears in the fourth summand. So $m_{0} \geq 3 n-3$ is sufficient. However, we already know that $m_{0}=$ $3 n-3$ fails, so $m_{0} \geq 3 n-2$.

\section{INJECTIONS}

The most natural proof of Conjecture 2.2 would be an injection from partitions of $k-1$ into partitions of $k$. We have found such injections for $n \leq 9$, but not for general $n$. One may also change Conjecture 2.2 to an equivalent injection on larger sets by using the $q$-binomial theorem. For example,

$$
\begin{aligned}
1-q+q^{n}+\sum_{k=2}^{\infty} & \frac{q^{k n}}{\prod_{i=2}^{k}\left(1-q^{i}\right)} \\
& =f_{n, m}(q)\left(1+\sum_{k=1}^{\infty} \frac{q^{k(m+1)}}{\prod_{i=1}^{k}\left(1-q^{i}\right)}\right) .
\end{aligned}
$$

An injection from the set representing the second term on the right side to the set for the left side is equivalent to Conjecture 2.2. One may hope that 
large values of $m$ would make an injection easier to find.

One may also begin with

$$
f_{n, n+1}(q)=\frac{1}{1-q^{n}}-\frac{q}{1-q^{n+1}},
$$

all of whose terms are known, and try adding $n+2$, $n+3, \ldots, 2 n-1$, to reach Conjecture 2.2. We use an injection to completely classify the negative terms for $f_{n, n+2}(q)$.

Theorem 4.1. Suppose $n=2 l+1>1$ is odd. All of the coefficients in $f_{n, n+2}(q)$ are at least -1 . Moreover the coefficient of $q^{k}$ is -1 if and only if

$$
k=a n+b(n+2)+1,
$$

where $0 \leq b=n j+r$ for $0 \leq r \leq l-1$, and $0 \leq a \leq a+j \leq r$.

For example, if $n=5$, then $r=0$ gives $k=1$, and $r=1$ gives $k=8,13,43$ as the four negative terms of $f_{5,7}(q)$.

Proof. We construct an injection from partitions of $k-1$ whose parts are from $\{n, n+1, n+2\}$, to partitions of $k$ whose parts are from $\{n, n+1$, $n+2\}$.

First, if $n+1$ is a part, add 1 to it to create a part of size $n+2$. So we assume $n+1$ is not a part, and the partition is $n^{a}(n+2)^{b}$. We need a partition of $a n+b(n+2)+1$ into parts of size $n$ and $n+1$.

Let $b=n j+r$, where $0 \leq r \leq n-1$. Define the injection by mapping $a n+b(n+2)+1$ to $n^{a+j+n-r}(n+1)^{n j+2(r-l)}$ if $r \geq l$, and to $n^{a+j-r-1} \times$ $(n+1)^{n j+2 r+1}$ if $0 \leq r \leq l-1$. It is routine to check the map is an injection where it is well defined. It is not well defined if and only if the multiplicity of $n$ in the second case is negative. These are the coefficients stated in Theorem 4.1, because they yield distinct integers for $a n+b(n+2)+1$.

\section{RELATED QUESTIONS}

It is natural to ask when $f_{n, n+m}(q) /(1-q)$ is strictly positive.
Theorem 5.1. There is an integer partition of $k$ into parts of size $\{n, n+1, \ldots, n+m\}$ for all

$$
k \geq n\left[\frac{n+m-2}{m}\right] .
$$

Moreover, this bound is best possible.

Proof. The $q$-binomial theorem implies, in terms of $q$-binomial coefficients,

$$
f_{n, n+m}(q) /(1-q)=\sum_{i=0}^{\infty}\left[\begin{array}{c}
m+i \\
i
\end{array}\right]_{q} q^{i n} .
$$

If $i \geq[(n+m-2) / m]$, the degree of the $q$-binomial coefficient is at least $n-1$, so all terms between $q^{i n}$ and $q^{(i+1) n-1}$ appear.

Friedman and Zeilberger [1993] proved that the coefficients of

$$
f_{2 n, 2 n+2 j}(q)(1-q)^{j}
$$

alternate in sign, so $P=\{2 n, \ldots, 2 n+2 j\}$ is asymptotically alternating. The next theorem classifies the asymptotically alternating sets $P$.

Theorem 5.2. $P=\left\{a_{1}, \ldots, a_{n}\right\}$ is asymptotically alternating if and only if $\alpha_{2} \geq \alpha_{j}$ for all $j$, where $\alpha_{j}$ is the number of indices $i$ such that $j \mid a_{i}$.

(As usual, $k \mid n$ means that $k$ divides $n$.)

Proof. We shall use the following fact from [Hardy et al. 1988]. If $p(q)$ is a real polynomial such that $p(0)=1$ and $p(q)>0$ for $q<0$, there exists an integer $a$ such that the coefficients of $(1-q)^{a} p(q)$ alternate in sign.

First assume that $\alpha_{2} \geq \alpha_{j}$ for all $j$. Let

$$
p(q)=\frac{(1-q)^{n-\alpha_{2}}\left(1-q^{a_{1} a_{2} \ldots a_{n}}\right)^{\alpha_{2}}}{\prod_{i=1}^{n}\left(1-q^{a_{i}}\right)} .
$$

It is easy to check using cyclotomic polynomials that $p(q)$ is a polynomial in $q$ with $p(0)=1$, and that $p(q)$ has no negative real roots. Thus there exists an integer $a>0$ such that $(1-q)^{a} p(q)$ is 
alternating, or equivalently $(1+q)^{a} p(-q)$ has nonnegative coefficients. Since $a_{1} a_{2} \ldots a_{n}$ is even, we see that

$$
\frac{(1+q)^{n-\alpha_{2}+a}}{\prod_{i=1}^{n}\left(1-(-q)^{a_{i}}\right)}
$$

has nonnegative coefficients. Replacing $q$ by $-q$ gives the first part of the theorem.

Next, suppose that $\alpha_{2}<\alpha_{j}$ for some $j$. We can assume that $\alpha_{j}$ is maximized, so that $j$ must be odd. We show that the coefficients cannot be alternating, by showing that the leading terms in the asymptotic expansion for the coefficients are not alternating for $a$ large.

The leading term in the partial fractions decomposition for the rational function is $A /(1-\omega q)^{\alpha_{j}}$, where $\omega$ is primitive $j$-th root of 1 , and

$$
A=\frac{\left(1-\omega^{-1}\right)^{a}}{\prod_{j \mid a_{i}} a_{i} \prod_{j \nmid a_{i}}\left(1-\omega^{-a_{i}}\right)} .
$$

The absolute value of the coefficient of $q^{k}$ is a polynomial in $k$, whose leading term is

$$
\left|\frac{A \omega^{k} k^{\alpha_{j}-1}}{\left(\alpha_{j}-1\right) !}\right|
$$

We first determine which primitive $j$-th roots $\omega$ maximize this quantity. Putting $\omega=\exp (2 \pi i m / j)$, we find

$$
|A|=c(2 \sin (\pi m / j))^{a},
$$

where $c$ is a constant independent of $a$. If $a$ is large enough, the largest value of $|A|$ occurs if $m=$ $\frac{1}{2}(j \pm 1)$, which is primitive. If there are many values of $j$ that maximize $\alpha_{j}$, the largest such $j$ with $m=\frac{1}{2}(j \pm 1)$ gives the largest value of $|A|$. Let $J$ denote the largest of these values of $j$.

Adding these two terms, we see that the sign of the coefficient of $q^{k}$, for large $k$, is the same as that of

$$
\cos \left(\frac{\pi k(J-1)}{J}+\varphi\right)
$$

where $\varphi$ is an angle independent of $k$. This implies that the sign behavior of the large coefficients is determined modulo $J$, not modulo 2 .

There is also a version of Theorem 5.2 that allows numerator factors. Odlyzko [1988] proved that the $k$-th difference for $P=\{1,2, \ldots\}$ is initially alternating, and then immediately nonnegative, for all large values of $k$.

\section{REFERENCES}

[Andrews 1976] G. E. Andrews, The Theory of Partitions, Encyclopedia of Mathematics and Its Applications 2, Addison-Wesley, Reading, MA, 1976 (reissued by Cambridge Univ. Press, 1985).

[Bateman and Erdös 1956] P. Bateman and P. Erdös, "Monotonicity of partition functions", Mathematika 3 (1956), 1-14.

[Friedman and Zeilberger 1993] J. Friedman and D. Zeilberger, "A generalization of Odlyzko's conjecture: the coefficients of $(1-q)^{j} /\left(\left(1-q^{2 n}\right) \ldots \times\right.$ $\left.\left(1-q^{2 n+2 j}\right)\right)$ alternate in sign", Proc. Amer. Math. Soc. 118 (1993), 1013.

[Hardy et al. 1988] G. Hardy, J. Littlewood, and G. Pólya, Inequalities, 2nd. ed., Cambridge Univ. Press, 1988.

[Odlyzko 1988] A. Odlyzko, "Differences of the partition function", Acta Arith. 49 (1988), 237-254.

Jane Friedman, Department of Mathematics, University of San Diego, San Diego, CA 92110 (janef@acusd.edu)

James T. Joichi, School of Mathematics, University of Minnesota, Minneapolis, MN 55455 (joichi@math.umn.edu)

Dennis Stanton, School of Mathematics, University of Minnesota, Minneapolis, MN 55455 (stanton@math.umn.edu)

Received March 7, 1994; accepted May 19 